ARCHIVO ESPAÑOL DE ARTE, LXXX, 320

OCTUBRE-DICIEMBRE 2007, pp. 423-444

ISSN: 0004-0428

\title{
VARIA
}

\section{LA FÁBRICA DE UCLÉS: LA GÉNESIS DEL PROYECTO Y LOS PRIMEROS AÑOS DE LAS OBRAS}

El convento de Uclés es uno de los conjuntos arquitectónicos más importantes de la Orden de Santiago. Su compleja historia constructiva ha despertado el interés de numerosos investigadores, como José María de Azcárate o María Luz Rokiski Lázaro. Azcárate fue el primero en utilizar los fondos documentales de la Orden de Santiago y, más concretamente, los libros de visita. Sus trabajos se centraron en el análisis de la primera fase de la obra, fase que comprendería el ala plateresca del convento y el comienzo de la iglesia. Además, aportó los primeros datos sobre Francisco de Luna, que en 1538 aparece citado como maestro prinçipal de la obra, y dio a conocer el nombre de varios canteros que trabajaron en la fábrica del convento ${ }^{1}$. María Luz Rokiski recogió las aportaciones de Azcárate y trazó un interesante bosquejo del desarrollo de su fábrica entre finales del siglo XVI y principios del XVII, momento en el que se construyó la mayor parte de la iglesia ${ }^{2}$. A pesar de estas aportaciones, la historia de este edifico presenta importantes lagunas que todavía no han sido aclaradas por los investigadores. La consulta de los fondos de la sección de Órdenes Militares del Archivo Histórico Nacional nos ha permitido localizar varios documentos de gran interés que contienen datos fundamentales sobre la génesis del proyecto $\mathrm{y}$, también, sobre el desarrollo de las obras durante sus cinco primeros años.

La construcción del nuevo convento fue un proyecto que tardó varios años en gestarse, barajándose distintas propuestas constructivas a lo largo de todo este tiempo. Las primeras gestiones de las que tenemos noticia se realizaron en 1525. Los miembros de la comunidad enviaron una petición al Consejo de Órdenes afirmando que la yglesya e casa del dicho convento es muy enferma e mal edificada e labrada e que en ella no ay sytio en que puedan bien edificar e labrar e tener aposento para todos los rreligiosos que conviene rresydir en el dicho convento ni para los comendadores e cavalleros de la dicha orden que van a estar en el ansy en provaçion e penitençia como a otras cosas ${ }^{3}$. Los religiosos querían acabar con estos problemas construyendo un nuevo edificio, pero no tenían

\footnotetext{
1 AzCÁRATe Ristori, José María de, "El convento de Uclés y Francisco de Luna, maestro de cantería”, Archivo Español de Arte, 115, 1956.

2 Rokiski Lázaro, María Luz, Arquitectura del siglo XVI en Cuenca, Cuenca, 1986.

3 AHN., OOMM., Archivo Histórico de Toledo, legajo 22.444, documento sin foliar (s.f.).
} 
la intención de levantar este recinto en el mismo lugar en el que estaba el antiguo, sino que pretendían trasladar la casa a otra parte que fuese mas sana. En concreto, habían pensado en una heredad propiedad del convento llamada Fuente Redonda que estaba situada a medio cuarto de legua de Uclés. Los religiosos solicitaron permiso para llevar a cabo el proyecto y, en respuesta a su petición, el Consejo de Órdenes emitió una provisión real dirigida a los visitadores generales de la Orden de Santiago en la provincia de Castilla. El documento, fechado el 7 de febrero de 1525, les encomendaba la misión de recabar información sobre el proyecto, información que debía ir acompañada de su propia opinión acerca del asunto.

El 9 de mayo el mayordomo del convento se personó en Villatobas, localidad donde estaban los visitadores, y les requirió con la provisión real, presentando al mismo tiempo un cuestionario que debía servir para entrevistar a los testigos que considerasen pertinentes. Los visitadores tomaron declaración a doce personas de la más diversa condición, entre los que se encontraban varios religiosos del convento, incluido el vicario; dos caballeros de la orden, los comendadores Diego de Torremocha y Bartolomé Mejía; el médico de la comunidad, el bachiller Juan de Cuenca; y el cirujano de la villa, maestre Lorenzo. Sus declaraciones corroboraron la versión que se ofrecía en la petición remitida al Consejo de Órdenes, aportando información adicional sobre las deficiencias del convento. Baste decir que la casa carecía de una enfermería y de una librería adecuadas y que no poseía sala capitular, por lo que los miembros de la comunidad se veían obligados a celebrar sus reuniones en el coro. Además, algún testigo, como el comendador Diego de Torremocha, afirmaba que en opinión de algunos maestros el convento carecía del arte (...) de jumetria (sic., geometría $)^{4}$.

A pesar del interés que tienen estas declaraciones, los testigos más importantes, al menos desde nuestro punto de vista, fueron los cuatro artistas que comparecieron para dar su opinión. Los visitadores se implicaron en el proyecto ya que eligieron personalmente a dos de ellos, en concreto a los más importantes: Antón Egas y Alonso de Covarrubias, que comparecieron en calidad de maestros de geometria. Los visitadores fueron muy claros al respecto, precisando que ellos habían hecho traer maestros jumetricos que (...) viesen el edificio ${ }^{5}$.

Antón Egas y Alonso de Covarrubias visitaron el convento, reconocieron cada una de sus estancias y diseñaron un nuevo edificio que trataba de dar respuesta a las necesidades de la comunidad. La descripción que realizó Antón del convento coincidía plenamente con los argumentos de la comunidad. Según sus propias palabras, la casa era estrecha de edifiçios e pobre dellos porque le faltan muchas pieças neçesarias ansy como yglesya e coro porque la que tienen es peque$\tilde{n} a$ e baxa e angosta e a lo que pareçe es ventosa e sera muy fria de ynvierno e desta cahusa tiene çerradas todas las ventanas e esta escura (sic.) e syn ninguna graçia. Además, el convento no tenía casa de capitulo ninguna ni enfermeria ni libreria ni çeldas prinçipales para rreligiosos viejos e otras personas rreverendas e las pieças que ay edificadas son pequeñas e mal moraderas por ser mal edificado como dicho es ${ }^{6}$.

La traza que realizaron Antón Egas y Alonso de Covarrubias incluía todos los añadimientos neçesarios para que la casa se pudiese morar e no ser tan dañosa a la salud de los que en ella bivieren e para que aya aposento conçertado e con cunplimientos para lo neçesario. Sin embargo, los maestros no tuvieron en cuenta los deseos de los religiosos, porque el proyecto

\footnotetext{
${ }^{4}$ AHN., OOMM., Archivo Histórico de Toledo, legajo 22.444, s.f.

5 Además en la declaración de Alonso de Covarrubias se aclara que éste fue llamado por los dichos visytadores juntamente con el dicho Anton Egas para ver la dicha casa e sytio. AHN., OOMM., Archivo Histórico de Toledo, legajo 22.444 , s.f.

${ }^{6}$ AHN., OOMM., Archivo Histórico de Toledo, legajo 22.444, s.f. Utilizamos la declaración de Antón Egas porque es la más extensa. Covarrubias se limitó a corroborar las opiniones de su compañero.
} 
que habían diseñado debía levantarse en el mismo lugar en el que se hallaba el antiguo convento y no en Fuente Redonda. Por eso, Antón también se preocupó de saber los fundamentos de donde se an de sacar las paredes desto que aqui se añade en la dicha traça, precisando que en la parte que menos hondo esta lo firme por los çimientos hasta ygualar con el suelo de la claostra deste dicho convento ay sesenta pies de hondo e en otras partes mas e para se edificar por la gran hondura de los çimientos rrequiere hazerse gran anchura de paredes e torres a trechos porque de otra manera no serian duraderos ni fuertes. Teniendo en cuenta todos estos datos, Antón Egas valoró el coste de lo que ansy se añade por la traça que agora se haze con los añadimientos en 32.600 ducados $^{7}$. En realidad, este proyecto no llegó a materializarse, pero lo cierto es que la existencia del diseño demuestra que la fábrica del convento estuvo vinculada desde un primer momento al foco constructivo toledano, y más concretamente a la familia Egas.

Los visitadores también recabaron la opinión de otros dos maestros que residían en la villa de Uclés: Francisco de Narváez y Juan Enriques, que comparecieron en calidad de maestros de carpintería y yesería. Ambos se limitaron a constatar las pésimas condiciones que reunía el edificio y a certificar los beneficios que se obtendrían con el traslado de la casa a la heredad de Fuente Redonda.

La propuesta de trasladar el convento despertó fuertes reticencias en el seno del Consejo de Órdenes. La opinión de los propios visitadores, que expresaron sus dudas acerca del proyecto en el pequeño informe que acompañaba a las declaraciones de los testigos, fue un elemento clave en todo este proceso, ya que sus argumentos tuvieron que influir de forma determinante en el parecer de los miembros del Consejo. Los visitadores se sintieron incapaces de emitir una opinión taxativa al respecto, pero lo cierto es que en su exposición de los hechos abundaban los argumentos en contra del traslado. En este sentido, afirmaban que la dicha casa e convento estando como esta edificada en lugar alto deve ser y es fria e ventosa de ynvierno mas de lo que seria estando en otro sytio llano pero que por estar el dicho sytio e casa fundada en alto alcança e tiene muy buenas vistas porque del mirador que tiene se vee e determina mucha tierra. De la misma forma, y aplicando una lógica aplastante, daban por sentado que las celdas de los religiosos, ubicadas en el ultimo piso, debían ser muy calurosas en verano, pero también se permitieron recordar que esto en cada parte e tierra lo ay ${ }^{8}$. El clima era algo que no se podía cambiar.

Los miembros de la comunidad eran conscientes de los obstáculos a los que se enfrentaban y, al final, se vieron obligados a cambiar de idea. Este cambio quedó reflejado en dos peticiones remitidas al Consejo por el prior del convento. En la primera, los religiosos solicitaban que el emperador mandase proveer como el dicho convento se traslade o se rreedifique donde esta. Hasta ese momento la comunidad seguía pensando que su proyecto era viable, pero en la segunda ya se había abandonado la idea de trasladar la casa y se optaba por levantar el nuevo edificio en el mismo lugar en el que se encontraba el antiguo. En la segunda petición, los religiosos hacían hincapié en la mucha neçesidad de aposentos que tenían y, como en la trasladacion del dicho convento se espera aver dilaçion, suplicaban al monarca que aya por bueno que se rrehedifique

\footnotetext{
${ }^{7}$ En relación con lo que costaría construir un convento de nueva planta en Fuente Redonda, Antón Egas precisó que el no sabía lo que querran labrar e edificar en el sytio nuevo e casa sy se trasladase pero que sy en la casa que se edificase de nuevo no se oviesen de hazer mas anchuras ni cunplimientos que en la casa que agora esta hecha e con lo traçado e añadido en ella por la traça nueva que en tal caso crehe e le pareçe que no costaria mas ni aun tanto hazerla de nuevo que aca con las enmiendas e añadido. A juicio de Antón Egas, las características orográficas del terreno sobre el que se levantaba el antiguo convento encarecerían enormemente la obra. AHN., OOMM., Archivo Histórico de Toledo, legajo 22.444, s.f.

8 AHN., OOMM., Archivo Histórico de Toledo, legajo 22.444, s.f.
} 
donde esta ${ }^{9}$. El cambio de postura debió facilitar los trámites, pero todavía tendremos que esperar hasta principios de 1529 para que comenzasen las obras.

Los datos que teníamos sobre los primeros años de la fábrica eran muy escasos, por no decir inexistentes, pero gracias a dos legajos del Archivo Histórico de Toledo hemos podido conocer el contenido de las cuentas del convento en el período comprendido entre finales de diciembre de 1527 y mediados de marzo de 1534 . El primer documento está fechado el 11 de marzo de 1531 e incluiría el período de sede vacante que se abrió el 27 de diciembre de 1527, período que terminó con la elección de Don Pedro García de Almaguer, más el trienio comprendido entre el 15 de marzo de 1528 y el 15 de marzo de $1531^{10}$. El segundo está fechado el 17 de marzo de 1534 y abarcaría el siguiente trienio, de marzo de 1531 a marzo de 153411.

El primer dato de interés que nos aporta el documento de 1531 está relacionado con el inicio de la obra. En las cuentas del primer trienio se afirma que la obra prinçipal comenzó el quinze de março de quinientos e veinte e nueve, fecha que difiere ligeramente de la que hasta ahora se venía manejando. Desde un punto de vista contable, el documento recoge, de una forma bastante genérica, todos los ingresos y gastos de la casa, pero lo que a nosotros nos interesa son los gastos del obrero, que se distribuyen en nueve apartados: yeso y agua, cal y arena, teja y ladrillo, madera, hierro y clavazón, jornales de oficiales, peones, destajos, piedra y gastos extraordinarios de obra. El capítulo más interesante es el que menciona las cantidades que cobraban los oficiales, capítulo que a pesar de no ser todo lo preciso que nosotros quisiéramos, nos aclara un aspecto clave de la historia del edificio, ya que recoge el nombre del maestro que realizó el proyecto definitivo. Según constaba en el libro del obrero, durante el segundo año del trienio se avian gastado en jornales de ofiçiales trezientas e diez e nueve mill e sieteçientos e ochenta e dos maravedis en çinco mill e ochoçientos e quarenta e nueve jornales y medio de ofiçiales que an entrado en la obra prinçipal este dicho año y en hazer la presa de Buenameson y en el molino de Miguel Arnaldos que se hizo de nuevo y con diez y nueve dias que se ocupo maestre Enrrique en hazer la traça y señalar la obra que ganava cada dia un castellano por mandado de su magestad ${ }^{12}$. Este maestre Enrique que aparece mencionado de forma tan escueta sería Enrique Egas. No es la primera vez que la documentación se limita a recoger su nombre o a mencionar su vinculación con la catedral de Toledo ${ }^{13}$; por eso no debemos extrañarnos ante la omisión del apellido. Por otra parte, el hecho de que la mención al maestro aparezca inmediatamente después de la referencia al molino de Miguel Arnaldos puede plantear problemas de interpretación en torno a la naturaleza de su labor. No obstante, si tenemos en cuenta la terminología empleada a lo largo del documento ${ }^{14}$, amén de otros factores como el tiempo invertido o la cantidad cobrada, no cabe ninguna duda al respecto. Enrique realizó la traza del convento y se encargó de marcar sobre el terreno las líneas de su diseño.

La vinculación que mantuvo Enrique Egas con la fábrica del convento no se limitó al diseño de su traza, sino que fue mucho más allá, ya que supervisó personalmente el desarrollo de la obra. Sin ir mas lejos entre los gastos del tercer año del trienio (marzo de 1530 a marzo de 1531) se

\footnotetext{
9 AHN., OOMM., Archivo Histórico de Toledo, legajo 22.444, s.f.

10 AHN., OOMM., Archivo Histórico de Toledo, legajo 8.385. Las cuentas fueron tomadas por Antonio de Valderravano, caballero de la orden.

11 AHN., OOMM., Archivo Histórico de Toledo, legajo 22.968. En esta ocasión, el encargado de tomar las cuentas fue Juan de Osorio.

12 AHN., OOMM., Archivo Histórico de Toledo, legajo 8.385 AHN., OOMM., Archivo Histórico de Toledo, legajo 8.385 , s.f.

13 AzCÁRAte Ristori, José María de, "Datos sobre las construcciones en el priorato de Uclés durante la primera mitad del siglo XVI", Boletín del Seminario de Estudios de Arte y Arqueología, 1959, pp. 109 y 122.

${ }^{14}$ La obra es una de las expresiones que se utiliza en las cuentas para hacer referencia a la fábrica del convento.
} 
incluyeron seys mill e sieteçientos e noventa maravedis que se dieron a maestre Enrrique por quatorze dias que handuvo visitando la obra a traçar cosas menudas a rrazon de un castillano (sic.) cada dia ${ }^{15}$.

Las cuentas del trienio comprendido entre marzo de 1531 y marzo de 1534 siguen el mismo esquema que las anteriores, pero en esta ocasión nos vamos a encontrar con datos de interés en dos apartados; aquel en el que se recogen los salarios que se pagaban a los maestros, apartado que ya hemos utilizado, y el destinado a los destajos, ya que nos servirá para conocer otros aspectos de la tarea desarrollada por los dos grandes maestros que estuvieron vinculados a la fábrica del convento: Enrique Egas y Francisco de Luna. Si repasamos el capítulo dedicado a los maestros nos volvemos e encontrar de forma reiterada con la figura de Enrique Egas. Durante el primer año del trienio se desembolsaron 459.356 maravedís para pagar los ocho mill e dozientos e sesenta e seys jornales de ofiçiales que se avian echado en la obra prinçipal deste convento y en las otras obras que ha tenido en Buenameson y Fuente Rredonda y en la casa de Ocaña y en otras lavores del dicho convento con nueve castellanos e medio que se dieron a maestre Enrrique de nueve dias e medio que vino a visitar la obra ${ }^{16}$. Las cuentas del siguiente año recogen el gasto de 438.548 maravedís en siete mill e nueveçientos e treynta e seys jornales de ofiçiales que andovieron en la obra del dicho convento a diversos preçios con quatro mill y ochoçientos e çinquenta maravedis que se dieron a maestre Enrrique por una vez que visito la obra este dicho año. De la misma forma, el último año se invirtieron otros 550.814 maravedís y medio en nueve mill e nueveçientos $e$ setenta e nueve jornales de ofiçiales que andovieron en la dicha obra a diversos preçios segund lo que cada uno meresçia y labrava e con çinco mill e trezientos e treynta e çinco maravedis que se dieron a maestre Enrrique por onze dias que se ocupo en la obra en el mes de setiembre ${ }^{17}$. A la vista de estas referencias parece obvio que Enrique Egas desempeñó un papel muy importante en el desarrollo de las obras. Sus periódicas visitas y, sobre todo, las enormes cantidades que cobraba nos estarían hablando de una labor muy importante; labor que, como ya señalamos anteriormente, estaría relacionada con la supervisión de la fábrica. La importancia que tenía la tarea desarrollada por Enrique Egas también queda de manifiesto en otro aspecto de la documentación que resulta muy significativo, ya que es el único maestro que aparece citado por su nombre en el apartado destinado a los gastos de los oficiales, consignándose además la cantidad que percibía por su trabajo.

El apartado destinado a los destajos también nos aporta información sobre los artífices que estaban vinculados al convento pero, a diferencia de lo que ocurría con los gastos de los maestros, este capítulo se limitaría a recoger la inversión realizada en aquellas obras que estaba llevando a cabo la comunidad en distintas heredades de su propiedad. Las cuentas del primer año lo dejan muy claro ya que según constaba en el libro del obrero se gastaron en obras e destajos fuera de la obra prinçipal 21.396 maravedís, cantidad que aparece desglosada de la siguiente forma: en la sierra del agua de Buenameson quinze ducados para el adobo della (...) y en seys ducados de rresta para acabar de pagar el destajo de la casa que se hizo en Ocaña y dos mill maravedis de çerrar unas vaderas en el molino de Mygarnaldos y en adobar un rrelox en la yglesia y ponello myll e dozientos e sesenta e un maravedis y ochenta rreales de quatro rrodeznos y veynte e dos rreales para unas puertas de la casa de Ocaña y veynte e quatro rreales de tres canales de Buenameson e mill e seteçientos maravedis de veynte tapias que se echaron en Fuente Rredonda ${ }^{18}$.

15 AHN., OOMM., Archivo Histórico de Toledo, legajo 8.385, s.f.

16 AHN., OOMM., Archivo Histórico de Toledo, legajo 22.968, s.f.

17 AHN., OOMM., Archivo Histórico de Toledo, legajo 22.968, s.f.

18 AHN., OOMM., Archivo Histórico de Toledo, legajo 22.968, s.f. El arreglo del reloj de la iglesia constituiría una pequeña excepción dentro de este apartado, ya que el elemento en cuestión estaría situado en el antiguo convento. 
Las partidas del segundo y tercer año del trienio nos muestran una realidad muy similar, mencionando distintas obras en varios molinos o en las casas que poseía el convento en Buenamesón, Ocaña o Santa María de los Llanos.

Determinar el contenido del apartado destinado a destajos puede parecer poco importante, pero la realidad es muy distinta porque, como ya hemos dicho, dentro de este capítulo figuran los nombres de dos maestros que también estaban vinculados a la fábrica del convento: Francisco de Luna y Enrique Egas. Francisco de Luna es el único que aparece mencionado todos los años: entre 1531 y 1532 cobró seis ducados, un año después recibió 7.750 maravedís y entre 1533 y 1534 recibió otros 10.000 maravedís. En el primero de los casos no se especifica la naturaleza del pago, pero en los otros dos se afirma que las cantidades se pagaban en concepto de salario. Enrique Egas sólo aparece en el apartado de destajos del último año del trienio, donde se consigna el pago de 6.000 maravedís que constituían la mitad del salario que debía recibir el maestro. Un salario que empezó a cobrar desde el día de Sant Miguell del año proximo pasado de treynta e tress ${ }^{19}$. La documentación no hace referencia al motivo concreto por el que percibían estos salarios pero, mientras no aparezcan datos que apunten en sentido contrario, parece lógico pensar que estaban relacionados con las tareas que desarrollaban en toda esa serie de obras menores que se estaban llevando a cabo en distintas posesiones del convento.

Los datos que acabamos de aportar tienen una gran importancia ya que resuelven determinadas incógnitas que hasta ahora carecían de respuesta, como la autoría del proyecto, y contribuyen a cambiar la visión que se tenía de otros aspectos, como la propia dirección de las obras. Las nuevas referencias documentales que hemos encontrado nos han desvelado el importante papel que desempeñó Enrique Egas, maestro que estuvo vinculado a la fábrica del convento prácticamente hasta la hora de su muerte. Él fue quién diseñó el proyecto y quién dirigió las obras, siendo bastante probable que a su fallecimiento fuera sustituido en esta tarea por Francisco de Luna que, como ya hemos dicho, aparece citado como maestro principal de la obra en 1538. Por otra parte, a la hora de valorar el papel que desempañaron estos dos maestros no podemos olvidarnos de la doble vinculación que mantuvieron con el convento; es decir, de su papel como maestros de la gran fábrica de Uclés y de su participación en esas otras obras que, en buena medida, estaban relacionadas con la arquitectura civil.

JoSÉ JAVIER BARRANQUERO CONTENTO

\section{FUENTES ICONOGRÁFICAS DE LA REPRESENTACIÓN DE CRISTO DEL RELIEVE DE LA ASCENSIÓN DEL CLAUSTRO DE SILOS}

No es algo novedoso señalar el hecho de la originalidad que presenta el relieve de la Ascensión del claustro románico de Santo Domingo de Silos a la hora de plasmar la figura de Jesús. El relieve, que es obra de finales del siglo XI del llamado primer maestro de Silos y se encuentra tallado en la cara interior izquierda del pilar sureste del claustro haciendo pendant con el relieve de Pentecostés, es en realidad la única Ascensión del arte antiguo y medieval donde Jesucristo es representado sólo por medio de su cabeza. Los autores que se han sucedido a lo largo de un siglo no han llegado a dar una explicación satisfactoria a este hecho, en el caso que se hayan preocupado de ello. El trabajo pues surge espontáneo de esta cuestión que al mirar cotidianamente el relieve nos está interrogando vehementemente. Y la respuesta surge, también espontánea, al hilo

${ }^{19}$ AHN., OOMM., Archivo Histórico de Toledo, legajo 22.968, s.f. 